\title{
Severe ketoacidosis in a non-diabetic lactating woman on a ketogenic diet
}

\author{
JOHN ALEXANDER, ${ }^{1}$ DINESH NAGI ${ }^{2}$
}

\begin{abstract}
Ketogenic diets are high fat, moderate protein, low carbohydrate diets with carbohydrate content usually less than $50 \mathrm{~g} /$ day. They are a novel intervention in the management of obesity and there is emerging evidence that they are very effective. Evidence regarding the long-term efficacy and safety of this rather new and popular intervention is still emerging and there is a lack of data on the effect of this diet in specific populations such as breast feeding women. We describe a case of severe ketoacidosis in a non-diabetic breast feeding woman who was successfully treated with conservative measures. This case highlights the need by medical and dietetic professionals for extra caution in initiating special dietary measures in susceptible physiological states.
\end{abstract}

Key words: diabetes mellitus, ketosis, ketoacidosis, ketogenic diet, non-diabetic ketoacidosis

\section{Introduction}

Ketogenic diets have gained a lot of attention in recent years as an effective intervention for weight loss. A ketogenic diet consists of low carbohydrate, high fat and adequate protein which in turn induces ketosis and promotes weight loss. This intervention has been established as a safe strategy in non-diabetic non-pregnant individuals. The experience of this diet among pregnant and lactating mothers is still minimal. We aim to draw the attention of the medical and dietetic fraternity to the risks involved in the introduction of a ketogenic diet in lactating women.

\section{Case presentation}

A healthy 22-year-old woman was referred to our local emergency department with shortness of breath, thirst and palpitations by her general practitioner. She did not report any symptoms of vomiting or diarrhoea and there were no symptoms suggestive of any

Specialist Registrar in Endocrinology and Diabetes, Mid Yorkshire NHS Hospitals Trust, Wakefield, UK

2 Consultant in Endocrinology and Diabetes, Mid Yorkshire NHS Hospitals Trust, Wakefield, UK

Address for correspondence: Dr John Alexander Specialist Registrar in Endocrinology and Diabetes, Edna Coates

Diabetes Centre, Pinderfields Hospital, Aberford Road,

Wakefield WF1 4DG, UK

E-mail: jonealexander@gmail.com

Br J Diabetes 2020;20:145-146

https://doi.org/10.15277/bjd.2020.255 systemic infection. She was 6 weeks post-partum and was breast feeding at the time. She was continuing on a normal diet until 3 days before presentation when she started on a ketogenic diet in order to attain weight loss after her pregnancy. Although she did not have any specialist dietetic advice, the diet she followed had less than $20 \mathrm{~g} /$ day of carbohydrate content. Her diet was guided by online information about ketogenic diets and she denied being on a starvation diet to attain weight loss. There were no concerns with the growth of the baby and no health issues in her baby were reported. Her past medical history included anaemia and mild intermittent asthma. She denied any use of alcohol or tobacco. Her regular medications included iron supplementation only. There was no family history of any autoimmune conditions such as type 1 diabetes and there was no previous history of mental health disorders or any eating disorders such as bulimia/anorexia nervosa. There was no known family history of any inborn errors of metabolism.

On arrival at the emergency department her observations showed a heart rate of 130 beats/min, blood pressure of 135/76 $\mathrm{mm} \mathrm{Hg}$, respiratory rate of $18 / \mathrm{min}$ and oxygen saturation of $100 \%$ on room air. Her temperature was $36.7^{\circ} \mathrm{C}$ and she looked comfortable. Her systemic examination was unremarkable except for slightly dry oral mucosa.

\section{Investigations}

The initial venous blood analysis revealed a pH of 7.05, base excess of 25.3, glucose of $4.2 \mathrm{mmol} / \mathrm{L}$, low bicarbonate and normal lactate. Her blood ketones were raised at $6.5 \mathrm{mmol} / \mathrm{L}$. All other investigations including full blood count, renal function and liver function were normal. Her inflammatory markers were normal. She also underwent a computed tomographic (CT) scan of the chest, abdomen and pelvis and a CT pulmonary angiogram.

\section{Differential diagnosis}

Extensive evaluation was done to look for causes of her severe acidosis and the CT scan of her chest, abdomen and pelvis did not reveal any abscess or pus collection. Her CT pulmonary angiogram done on arrival at the hospital ruled out any pulmonary embolism.

\section{Treatment}

Her initial diagnosis was ketogenic diet and lactation-induced ketoacidosis. She was admitted to the ward and started on intravenous fluid replacement with $0.9 \%$ sodium chloride solution along with a normal carbohydrate-rich diet. She responded well to the treatment. 


\section{Outcome and follow-up}

Her repeat pH after 24 hours was 7.35 and base excess improved to -3.9 . She remained euglycaemic with normal blood glucose throughout her stay. Her $\mathrm{HbA}_{1 \mathrm{c}}$ has remained normal even after 1 year of close follow-up. We believe that her final diagnosis on the basis of the above findings was ketogenic diet-induced ketoacidosis.

\section{Discussion}

Ketoacidosis has been described in relation to multiple conditions including diabetic ketoacidosis, alcoholic ketoacidosis, starvation ketoacidosis and various rare forms of inborn errors of metabolism such as succinyl CoA:3 ketoacid CoA transferase deficiency, mitochondrial 2-methylacetoacetyl COA thiolase deficiency and methylmalonyl-CoA mutase deficiency. ${ }^{1}$ Mortality remains high if this condition is not treated promptly. There is evidence in the veterinary literature about the occurrence of ketoacidosis in lactating cattle, especially when the dietary substrate was not enough to compensate for the high metabolic demand of lactation. ${ }^{2}$ However, the incidence of this condition in human beings has been rarely reported. Although a few cases of lactation ketoacidosis have been described, ${ }^{3-5}$ our index case describes the severity and rapid onset of the ketoacidosis that can develop in a lactating female on a ketogenic diet.

Ketogenic diets have been proven to have a beneficial effect in certain conditions such as uncontrolled epilepsy ${ }^{6}$ and obesity. They are also being tried in different medical conditions such as type 2 diabetes, polycystic ovarian syndromes and malignancy. ${ }^{7}$ The effect of a ketogenic diet on the metabolic parameters in pregnancy and lactation is not well established. A low carbohydrate diet would burn the body fat as a primary source of energy by converting it to fatty acids and subsequently to ketone bodies. Although there is an increase of ketones in the blood, it is unlikely that these high levels would cause any clinically significant changes in the acid-base status in normal individuals. ${ }^{8,9}$ High metabolic states like lactation would need more substrates for energy, and a ketogenic diet in those situations could lead to depletion of glycogen stores, subsequently leading to excess ketone production to the extent that it upsets the acid-base balance. This appears to be the causative factor in our case.

The diagnosis can sometimes be delayed due to the fact that ketosis may not be suspected in a non-diabetic patient with normal glucose levels. There was no history suggestive of starvation, alcoholism or diabetes in our case, hence ruling out the commonly occurring causes of ketoacidosis. Euglycemic diabetic ketoacidosis is a condition similar to the presentation described here, but is typically seen in either diagnosed or undiagnosed diabetic patients in relation to intercurrent illnesses or with use of medications like sodium-glucose co-transporter-2 (SGLT2) inhibitors. They require aggressive fluid resuscitation along with use of intravenous insulin infusion. ${ }^{10}$ Reassuringly, our patient responded quickly to the conservative management and reintroduction of a normal carbohydrate diet. Previously reported cases of ketogenic diet-induced ketoacidosis were treated with intravenous glucose with intravenous insulin, although our case

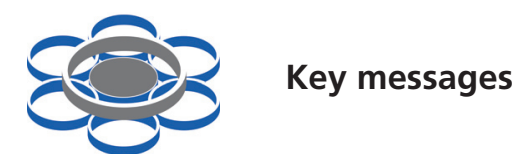

- Although a ketogenic diet has been publicised as a successful weight loss modality, it is unclear about its safety during lactation

- Any intervention to achieve a patient's pre-pregnancy weight should be discussed with medical professionals

- Our case report aims to increase the awareness regarding the incidence of ketoacidosis among lactating women on a ketogenic diet

responded well to just $0.9 \%$ sodium chloride solution and a normal diet. The normal blood glucose levels and normal $\mathrm{HbA}_{1 c}$ one year after the episode rules out diabetes mellitus as a cause of her ketoacidosis.

It is important to be aware that weight loss strategies should be catered to the metabolic demands of the body at the time. Multiple anecdotal evidence from published case reports, $, 4,6$ including ours, suggest that a ketogenic diet during lactation can sometimes be life-threatening.

Patient's perspective The patient has been under follow-up and she is quite happy with the treatment she received.

\section{Conflict of interest None. \\ Funding None.}

\section{References}

1. Gleeson S. Lactation ketoacidosis: an unusual entity and a review of the literature. Perm J 2016;20(1):71-3. https://doi.org/10.7812/TPP/15-097

2. Holtenius $P$, Holtenius $K$. New aspects of ketone bodies in energy metabolism of dairy cows: a review. Zentralb/ Veterinarmed A 1996;43(110):579-87. https://doi.org/10.1111/j.1439-0442.1996.tb00491.x

3. von Geijer L, Ekelund M. Ketoacidosis associated with low-carbohydrate diet in a non-diabetic lactating woman: a case report. J Med Case Rep 2015;9:224. https://doi.org/10.1186/s13256-015-0709-2

4. Nnodum B, Oduah E, Albert D, Pettus M. Ketogenic diet-induced severe ketoacidosis in a lactating woman: a case report and review of the literature. Case Rep Nephro/ 2019;2019:1214208. https://doi.org/10.1155/ 2019/1214208

5. Basnet S, Tachamo N, Nazir S, Dhital R, Jehangir A, Donato A. Severe anion gap metabolic acidosis associated with initiation of a very lowcarbohydrate diet. J Community Hosp Intern Med Perspect 2019; 9(2):165-7. https://doi.org/10.1080/20009666.2019.1583534

6. Vining E. A multicenter study of the efficacy of the ketogenic diet. Arch Neurol 1998;55(11):1433-7. https://doi.org/10.1001/archneur.55.11.1433

7. Paoli A, Rubini A, Volek J, Grimaldi K. Beyond weight loss: a review of the therapeutic uses of very-low-carbohydrate (ketogenic) diets. Eur J Clin Nutr 2013;67(8):789-96. https://doi.org/10.1038/ejcn.2013.116

8. Gomez-Arbelaez D, Crujeiras A, Castro A, et al. Acid-base safety during the course of a very low-calorie-ketogenic diet. Endocrine 2017; 58(1):81-90. https://doi.org/10.1007/s12020-017-1405-3

9. Yancy W, Olsen M, Dudley T, Westman E. Acid-base analysis of individuals following two weight loss diets. Eur J Clin Nutr 2007;61(12):141622. https://doi.org/10.1038/sj.ejcn.1602661

10. Barski L, Eshkoli T, Brandstaetter E, Jotkowitz A. Euglycemic diabetic ketoacidosis. Eur J Intern Med 2019;63:9-14. https://doi.org/10.1016/ j.ejim.2019.03.014 